\title{
Riesgos psicosociales en el trabajo en colaboradores de una empresa minera de Arequipa.
}

Psychosocial risks at work in employees of a mining company in Arequipa.

José Manuel Calizaya López, Felipe Mario Zapata Delgado y José Luis Evangelista Aliaga

Universidad Nacional de San Agustín. Arequipa Perú.

\section{INFORMACIÓN}

\section{Historia del Artículo}

Recepción: 13/01/2020

Revisión: 12/03/2020

Aceptación: 20/05/2020

\section{Palabras Clave}

Riesgos psicosociales, exposición al riesgo, colaboradores

\section{Key Words}

Psychosocial risks, risk exposure, collaborators

\section{DOI}

https://doi.org/10.35286/veritas. v21i 2.274

\begin{abstract}
RESUMEN
La presente investigación planteó como objetivo medir la situación de exposición a riesgos psicosociales en el trabajo en colaboradores según variables sociodemográficas en una empresa minera de Arequipa. Se utilizó el cuestionario de autoevaluación de riesgos psicosociales en el trabajo COPSOQ - ISTAS 21, versión 1.5, en una muestra de 201 colaboradores, el estudio utilizó el diseño metodológico cuantitativo, descriptivo - comparativo, transversal. El análisis estadístico describió que los datos no presentan distribución normal, es así que de acuerdo a los resultados se demostró que el nivel de exposición a riesgos psicosociales es desfavorable siendo los colaboradores obreros en comparación a los empleados y funcionarios quienes presentan mayores problemas en las exigencias psicológicas, control sobre el trabajo, apoyo social, doble presencia y estima, así también, según sexo son las mujeres quienes presentan mejores resultados en las dimensiones citadas debido a que controlan su trabajo, reciben apoyo de compañeros y superiores, y el trato es adecuado en comparación a los varones, y las correlaciones sobre las exigencias psicológicas demostraron que existe alta probabilidad de perjudicar la salud física, mental y social del colaborador.
\end{abstract}

\begin{abstract}
The objective of this research was to measure the situation of exposure to psychosocial risks at work in collaborators according to sociodemographic variables in a mining company in Arequipa. The psychosocial risk self-assessment questionnaire was used in the COPSOQ - ISTAS 21 work, version 1.5 , in a sample of 201 collaborators, the study used the quantitative, descriptive - comparative, cross-sectional methodological design. The statistical analysis described that the data do not present a normal distribution, so, according to the results, it was shown that the level of exposure to psychosocial risks is unfavorable, being the worker collaborators in comparison to the employees and officials who present greater problems in the demands. psychological, control over work, social support, double presence and esteem, as well as, according to sex, women have the best results in the dimensions mentioned because they control their work, receive support from colleagues and superiors, and the treatment is appropriate Compared to men, and the correlations on the psychological demands showed that there is a high probability of damaging the physical, mental and social health of the collaborator.
\end{abstract}

\section{INTRODUCCIÓN}

Los riesgos psicosociales son situaciones adversas a los que están expuestos los colaboradores de cualquier organización (Bravo \& Nazar, 2015), el estudio presenta relevancia científica para la gestión de recursos humanos debido a la importancia de la seguridad integral que debe primar en el ambiente laboral, valorando no solo la salud física y el ambiente sino también la salud mental y social del colaborador (Moreno, 2011).

En ese sentido, los procesos laborales y el diseño del trabajo en las organizaciones han cambiado en los últimos años generando nuevos riesgos psicosociales que afectan la salud y la calidad de vida laboral (Gil, 2012), el nivel de exposición a estos riesgos puede generar altos niveles de

Correspondencia:

José Manuel Calizaya López

jcalizayal@unsa.edu.pe estrés, produciendo situaciones negativas que repercuten en la productividad, el clima, las relaciones interpersonales de los colaboradores (Camacho \& Mayorga, 2017).

De igual manera, de acuerdo a la Ley 29783 (Ley de Seguridad y Salud en el Trabajo) reconoce la existencia de riesgos psicosociales en el ámbito laboral, por lo que, las condiciones de trabajo (Castro, 2018) es un factor importante a tomar en cuenta, debido a que la responsabilidad empresarial debe velar por la seguridad, la salud, la higiene y el ambiente laboral (Moreno, 2011; Gómez, Hernández, \& Méndez, 2014), situación que también es considerada como un riesgo por la poca atención que presta la mayoría de empresas en nuestro país.

Además, que el horario y turno de trabajo también inciden en la exposición de riesgos psicosociales al interior de las organizaciones debido a que pueden generar enfermedades profesionales y accidentes de trabajo, repercutiendo en la salud integral del colaborador (Varela, 2014).

En relación, a la información estadística proporcionada por el Ministerio de Trabajo (2019) solo se evidencian datos estadísticos sobre notificaciones de accidentes de 
trabajo, incidentes peligrosos y enfermedades ocupacionales, al respecto, a diciembre del 2019, se registraron 2763 notificaciones de accidentes de trabajo de un total de 1616 empresas, representando el $97.18 \%$ a accidentes de trabajo, $0.90 \%$ accidentes mortales, $1.88 \%$ incidentes peligrosos, y $0.04 \%$ enfermedades ocupacionales, siendo la industria manufacturera $(22.01 \%)$ la actividad económica que tuvo mayor cantidad de notificaciones; así mismo, el Ministerio de Energía y Minas reveló a diciembre del 2019, que las principales enfermedades ocupacionales que afectan la salud de los colaboradores en los centros mineros son: hipoacusia neurosensorial bilateral $(23.08 \%)$, hipoacusia mixta conductual neurosensorial bilateral (30.77\%), neumoconiosis debida a polvos que contienen sílice $(38.46 \%)$ y efecto toxico de otras sustancias inorgánicas magnesio y sus compuestos (7.69\%), evidenciándose que no se ha registrado información sobre problemas de salud mental y social en el trabajo como: estrés laboral, desmotivación, ansiedad, depresión, insatisfacción laboral, síndrome de burnout, interacción social, moobing o acoso laboral y karoshi (Martínez \& Hernández, 2005; Luna, Anaya, \& Ramírez, 2019).

De otro lado, los riesgos psicosociales también se diferencian por variables sociodemográficas (Gil, 2012), en ese sentido, se han encontrado estudios que miden el nivel de exposición a riesgos psicosociales en el trabajo según sexo y edad; en relación al sexo son las mujeres que se encuentran menos expuestas a riesgos que los varones debido a que, por lo general, la mujer realiza actividades administrativas y se encuentra en oficinas mientras que el varón realiza trabajos en campo sobre todo dependiendo del sector empresarial; con respecto a la edad son los adultos de 55 años a más que están expuestos a riesgos que los jóvenes menores de 30 años, es decir a mayor edad mayor exposición a riesgos psicosociales (ISTAS-CCOO, 2019; Tacca \& Tacca, 2019).

En referencia a las dimensiones de estudio de riesgos psicosociales el presente estudio toma en consideración las exigencias psicológicas (cantidad de trabajo), el control sobre el trabajo (autonomía para realizar el trabajo), la doble presencia (demandas del trabajo y las actividades domésticas juntas), el apoyo social y la calidad de liderazgo (apoyo de superiores, compañeros y la definición de tareas), la estima (el trato que se recibe en el trabajo) y la inseguridad sobre el futuro (preocupación por los cambios o perdida de trabajo), (Evaluación de Riesgos Psicosociales en el Trabajo CoPsoQistas, 2010).

Por lo tanto, de acuerdo a lo descrito la investigación planteó como objetivo medir la situación de exposición a riesgos psicosociales en el trabajo en colaboradores según variables sociodemográficas en una empresa minera de Arequipa.

\section{MATERIAL Y MÉTODOS}

\section{Instrumento}

Para medir la situación a exposición de riesgos psicosociales, se utilizó el cuestionario de autoevaluación de riesgos psicosociales en el trabajo COPSOQ - ISTAS 21 , versión 1.5 , que es la adaptación del Cuestionario Psicosocial de Copenhague a la realidad del mercado de trabajo y relaciones laborales en trabajadores Españoles; el instrumento consta de 38 ítems de respuesta tipo Likert, las puntuaciones de los ítems varían de acuerdo a la situación ( siempre $=4$, muchas veces $=3$, algunas veces $=2$, solo alguna vez $=1$, nunca $=0$ ) y viceversa, la escala se distribuye en 6 dimensiones con sus respectivos puntos de corte: Dimensión 1: exigencias psicológicas (6 ítems, favorable 0-7, intermedia 8-11, desfavorable 12-24), dimensión 2: control sobre el trabajo (10 ítems, favorable 26-40, intermedia 19 a 25, desfavorable 0-18), dimensión 3: inseguridad sobre el futuro (4 ítems, favorable 0-4, intermedia 5-9, desfavorable 10-16), dimensión 4: apoyo social y calidad de liderazgo (10 ítems, favorable 32-40, intermedia 25-31, desfavorable $0-24$ ), dimensión 5: doble presencia (4 ítems, favorable 0-2, intermedia 3-6, desfavorable 7-16), y dimensión 6: estima (4 ítems, favorable 13-16, intermedia 10-12, desfavorable 0-9). Así mismo, en el instrumento se adicionaron características sociodemográficas como edad, sexo y condición laboral; de igual manera se consignaron los datos del consentimiento informado.

Para la versión adaptada a la muestra local, se obtuvieron los niveles de confiabilidad a través de la prueba alfa de Cronbach, tal como se describe en la tabla 1, por lo tanto, el instrumento posee buena confiabilidad cuando los valores (alfa) $\alpha \geq 0.700$ (Frias, 2019).

Tabla 1: Índices de confiabilidad del instrumento COPSOQ - ISTAS 21, versión 1.5

\begin{tabular}{ccc}
\hline & \multicolumn{2}{c}{ Índices de confiabilidad } \\
\hline Riesgos psicosociales & $\mathrm{N}$ & $\alpha$ \\
\hline Escala general & 201 & 0.867 \\
Exigencias psicológicas & 201 & 0.896 \\
Control sobre el trabajo & 201 & 0.815 \\
Inseguridad sobre el futuro & 201 & 0.911 \\
Apoyo social y calidad del liderazgo & 201 & 0.821 \\
Doble presencia & 201 & 0.815 \\
Estima & 201 & 0.821 \\
\hline
\end{tabular}

Nota: $\mathbf{N}=$ Muestra; $\boldsymbol{\alpha}=$ alfa de Cronbach.

\section{Diseño}

La investigación utilizó el diseño metodológico cuantitativo, descriptivo - comparativo, transversal, para determinar la situación de exposición a los riesgos psicosociales según sexo, edad y condición laboral en la muestra seleccionada.

Participantes

La muestra seleccionada para la ejecución de la investigación fueron 201 colaboradores de una empresa privada de Arequipa, que desarrolla actividades de explotación minera, la muestra fue seleccionada aleatoriamente. En relación a la edad promedio de los colaboradores fue de 36 años con una desviación de \pm 8.06 dentro de un rango de 21 a 66 años. Con respecto al sexo de los colaboradores el 5.5\% fueron mujeres y el $94.5 \%$ varones. Así también, el $6.5 \%$ de los colaboradores presentaron la condición laboral de funcionarios, $25.4 \%$ empleados y $68.2 \%$ obreros. 


\section{Procedimiento}

Se aplicó el instrumento a los colaboradores previa autorización en las diferentes zonas de trabajo después de la jornada laboral, se les informó sobre la investigación para que expresen su voluntad de participar a través del consentimiento informado. Para el análisis estadístico se utilizó el programa SPSS 25.0. La exploración de la normalidad de los datos a través de la prueba de KolmogorovSmirnov (Yazici \& Yolacan, 2007), demostró que no existe distribución normal, por lo tanto, se utilizaron estadísticos no paramétricos. Se realizó el análisis categórico de la situación de exposición a riesgos psicosociales y sus dimensiones; para encontrar diferencias significativas según características sociodemográficas, se utilizaron los estadígrafos $U$ de Mann-Whitney (para dos muestras independientes), la $\mathrm{H}$ de Kruskall Wallis (para más de dos muestras independientes) y para establecer correlaciones se utilizó la Rho de Spearman.

\section{RESULTADOS}

En la tabla 2, se describe los valores numéricos de la variable riesgos psicosociales para medir la situación a la que se encuentran expuestos los colaboradores, en ese sentido, el $50 \%$ de los colaboradores presenta puntajes menores a 56 , de un puntaje máximo de 134 , demostrándose que el puntaje es desfavorable lo que indica que la mayoría de colaboradores se encuentran expuestos a riesgos psicosociales en el trabajo.

Tabla 2: Análisis descriptivo numérico de la variable riesgos psicosociales

\begin{tabular}{cccccc}
\hline & M & DE & Md & Min. & Max. \\
\hline Riesgos psicosociales & 68.7 & 32.4 & 56.0 & 19 & 134 \\
\hline
\end{tabular}

Nota: $M=$ media aritmética; $\mathbf{D E}=$ desviación estándar $; \mathbf{M d}=$ mediana $;$ Min $=$ puntaje mínimo; Max = puntaje máximo

En la tabla 3, se describe los valores categóricos de las dimensiones de la variable riesgos psicosociales, demostrándose que: En relación a las exigencias psicológicas el $98 \%$ de colaboradores indican que el nivel de exposición psicosocial es desfavorable para la salud por la cantidad de trabajo; con respecto al control sobre el trabajo el $56.7 \%$ precisa que el nivel de exposición es desfavorable, $28.9 \%$ es favorable y $14.4 \%$ señala que es intermedia; en cuanto a la inseguridad sobre el futuro en el trabajo el $80.1 \%$ considera que es desfavorable, $14.4 \%$ es de nivel intermedia y sólo el $5.5 \%$ de colaboradores indican que se sienten seguros en su trabajo (favorable); en relación al apoyo social y calidad de liderazgo según los colaboradores el 71.6\% menciona que el nivel de exposición es desfavorable para la salud debido a que perciben que no reciben integralmente el apoyo de sus superiores y compañeros, el $20.9 \%$ considera que el nivel es intermedio y el $7.5 \%$ precisa que si recibe ese apoyo social; según la dimensión doble presencia (respuesta simultánea a las demandas del trabajo y las actividades domésticas), el $43.8 \%$ considera que el nivel de exposición es intermedia, $29.4 \%$ es desfavorable y $26.9 \%$ considera que es favorable; en cuanto a la dimensión estima (trato recibido en el trabajo) el $71.6 \%$ señala que es desfavorable para la salud (no reciben un adecuado trato), $20.9 \%$ el nivel es intermedia y $7.5 \%$ considera que es favorable.

Tabla 3: Análisis categórico de los riesgos psicosociales según dimensiones

\begin{tabular}{cccccccc}
\hline & \multicolumn{2}{c}{ Desfavorable } & \multicolumn{2}{c}{ Intermedio } & \multicolumn{2}{c}{ Favorable } \\
\hline Riesgos psicosociales & $\mathrm{f}(\mathrm{x})$ & $\%$ & $\mathrm{f}(\mathrm{x})$ & $\%$ & $\mathrm{f}(\mathrm{x})$ & $\%$ \\
\hline Exigencias psicológicas & 197 & 98.0 & 4 & 2.0 & 0 & 0.0 \\
Control sobre el trabajo & 114 & 56.7 & 29 & 14.4 & 58 & 28.9 \\
$\begin{array}{c}\text { Inseguridad sobre el futuro } \\
\begin{array}{c}\text { Apoyo social y calidad del } \\
\text { liderazgo }\end{array}\end{array}$ & 161 & 80.1 & 29 & 14.4 & 11 & 5.5 \\
$\begin{array}{c}\text { Doble presencia } \\
\text { Estima }\end{array}$ & 59 & 29.4 & 88 & 43.8 & 54 & 26.9 \\
\hline
\end{tabular}

Nota: $f(x)=$ frecuencia; $\%=$ porcentaje

Al comparar las dimensiones de la variable riesgos psicosociales según sexo (tabla 4), se observa que existen diferencias estadísticamente significativas en la mayoría de dimensiones a excepción de la dimensión inseguridad sobre el futuro en el trabajo, siendo los hombres quienes mayormente están expuestos a exigencias psicológicas que las mujeres; y son las mujeres quienes tienen mejores posibilidades de control sobre el trabajo reciben apoyo de los superiores y compañeros, manejan adecuadamente sus actividades labores con las domesticas (más organizadas) y reciben mejor trato en comparación que los varones.

Tabla 4: Prueba U de las dimensiones de la variable riesgos psicosociales según sexo

\begin{tabular}{ccccc}
\hline & Hombres & Mujeres & & \\
\cline { 2 - 5 } Dimensiones & $(\mathrm{n}=190)$ & $(\mathrm{n}=117)$ & & $\mathrm{p}$ \\
\cline { 2 - 5 } & $\mathrm{Md}$ & $\mathrm{Md}$ & $\mathrm{U}$ & 0.049 \\
\hline $\begin{array}{c}\text { Exigencias } \\
\text { psicológicas }\end{array}$ & 102.9 & 68.3 & 685.5 & 0.006 \\
$\begin{array}{c}\text { Control sobre el } \\
\text { trabajo }\end{array}$ & 98.3 & 147.2 & 536.5 & \\
$\begin{array}{c}\text { Inseguridad sobre el } \\
\text { futuro }\end{array}$ & 100.2 & 115.2 & 890.0 & 0.392 \\
$\begin{array}{c}\text { Apoyo social y } \\
\text { calidad del liderazgo }\end{array}$ & 98.5 & 144.8 & 563.0 & 0.008 \\
$\begin{array}{c}\text { Doble presencia } \\
\text { Estima }\end{array}$ & 98.6 & 143.3 & 579.5 & 0.011 \\
\hline
\end{tabular}

Nota: $\mathbf{n}=$ tamaño muestral; $M d$ = Mediana; $\mathrm{U}=\mathbf{U}$ de Mann-Whitney; $\mathbf{p}=\mathbf{p}$ valor.

En la tabla 5, se compararon las dimensiones de la variable riesgos psicosociales según condición laboral, demostrando que existen diferencias estadísticamente significativas en la mayoría de dimensiones a excepción de las dimensiones exigencias psicológicas e inseguridad sobre el futuro en el trabajo. Observándose que son los colaboradores en condición de funcionarios de la empresa 
quienes presentan mejor control sobre su trabajo, reciben el apoyo social de los demás colaboradores, manejan la doble presencia y consideran que reciben el mejor trato en la empresa en comparación a los colaboradores empleados y obreros.

Tabla 5: Prueba $\mathrm{H}$ de las dimensiones de la variable riesgos psicosociales según condición laboral

\begin{tabular}{cccccc}
\hline & Funcionario & Empleado & Obrero & & \\
\cline { 2 - 6 } Dimensiones & $(\mathrm{n}=13)$ & $(\mathrm{n}=51)$ & $(\mathrm{n}=137)$ & & \\
\cline { 2 - 6 } & $\mathrm{Md}$ & $\mathrm{Md}$ & $\mathrm{Md}$ & $\mathrm{H}$ & $\mathrm{p}$ \\
\hline $\begin{array}{c}\text { Exigencias psico- } \\
\text { lógicas }\end{array}$ & 95.3 & 99.1 & 102.3 & 4.3 & 0.120 \\
$\begin{array}{c}\text { Control sobre el } \\
\text { trabajo }\end{array}$ & 172.5 & 162.0 & 71.5 & 140.4 & 0.000 \\
$\begin{array}{c}\text { Inseguridad sobre } \\
\text { el futuro }\end{array}$ & 88.7 & 109.4 & 99.0 & 3.8 & 0.153 \\
$\begin{array}{c}\text { Apoyo social y ca- } \\
\text { lidad del liderazgo }\end{array}$ & 180.9 & 146.3 & 76.6 & 127.7 & 0.000 \\
$\begin{array}{c}\text { Doble presencia } \\
\text { Estima }\end{array}$ & 172.0 & 153.3 & 74.8 & 101.5 & 0.000 \\
\hline
\end{tabular}

Nota: $\mathbf{n}=$ tamaño muestral; $M d=$ Mediana; $\mathbf{H}=\mathbf{H}$ de Kruskal Wallis; $\mathbf{p}=\mathbf{p}$ valor.

Al comparar las dimensiones de la variable riesgos psicosociales según edad (tabla 6), se observa que existen diferencias estadísticamente significativas sólo en las dimensiones exigencias psicológicas y doble presencia, en el resto de dimensiones no existe diferencias. En relación a la dimensión exigencias psicológicas son los colaboradores mayores de 31 a 66 años quienes consideran que la cantidad de trabajo afecta desfavorablemente la salud en comparación a los colaboradores menores de 30 años y en la dimensión doble presencia son los colaboradores que presentan edades entre 31 a 40 años quienes manejan mejor las actividades laborales y domesticas en comparación a los otros colaboradores según edad.

Tabla 6: Prueba $\mathrm{H}$ de las dimensiones de la variable riesgos psicosociales según edad

\begin{tabular}{ccccccc}
\hline & $\begin{array}{c}21 \text { a } 30 \\
\text { años }\end{array}$ & $\begin{array}{c}31 \text { a } 40 \\
\text { años }\end{array}$ & $\begin{array}{c}41 \text { a } 50 \\
\text { años }\end{array}$ & $\begin{array}{c}\text { Más de } 51 \\
\text { años }\end{array}$ & & \\
\cline { 2 - 7 } Dimensiones & $(\mathrm{n}=45)$ & $(\mathrm{n}=91)$ & $(\mathrm{n}=56)$ & $(\mathrm{n}=9)$ & & \\
\cline { 2 - 7 } & $\mathrm{Md}$ & $\mathrm{Md}$ & $\mathrm{Md}$ & $\mathrm{Md}$ & $\mathrm{H}$ & $\mathrm{p}$ \\
\hline $\begin{array}{c}\text { Exigencias } \\
\text { psicológicas }\end{array}$ & 94.1 & 103.0 & 103.0 & 103.0 & 14.1 & 0.003 \\
$\begin{array}{c}\text { Control sobre el } \\
\text { trabajo }\end{array}$ & 107.9 & 106.2 & 86.5 & 103.8 & 6.2 & 0.103 \\
$\begin{array}{c}\text { Inseguridad sobre } \\
\text { el futuro }\end{array}$ & 104.9 & 103.4 & 97.9 & 76.6 & 4.4 & 0.224 \\
$\begin{array}{c}\text { Apoyo social } \\
\text { y calidad del } \\
\text { liderazgo }\end{array}$ & 100.0 & 104.3 & 95.5 & 106.7 & 1.4 & 0.695 \\
$\begin{array}{c}\text { Doble presencia } \\
\text { Estima }\end{array}$ & 102.4 & 110.3 & 86.4 & 91.4 & 7.0 & 0.041 \\
\hline
\end{tabular}

Nota: $\mathbf{n}=$ tamaño muestral; $M d=$ Mediana; $\mathbf{H}=\mathbf{H}$ de Kruskal Wallis; $\mathbf{p}=\mathbf{p}$ valor
A nivel correlacional, se observa que existe relación entre la variable riesgos psicosociales y la mayoría de dimensiones a excepción de la inseguridad sobre el futuro en el trabajo; las correlaciones en las dimensiones control sobre el trabajo, apoyo social y calidad de liderazgo, doble presencia y estima son positivas estadísticamente significativas; $y$ con respecto a las exigencias psicológicas y los riesgos psicosociales la correlación es negativa (inversa), existiendo la probabilidad que a mayor exposición psicosocial es menos favorable para la salud (existe alta probabilidad de perjudicar la salud).

Tabla 7: Correlaciones de los riesgos psicosociales y las dimensiones de estudio

\begin{tabular}{ccc}
\hline & \multicolumn{2}{c}{$\begin{array}{c}\text { Riesgos psicosociales } \\
\text { (R de Spearman) }\end{array}$} \\
\cline { 2 - 3 } Dimensiones & $\mathrm{r}$ & $\mathrm{p}$ \\
\hline Exigencias psicológicas & $-0.426^{* *}$ & 0.000 \\
Control sobre el trabajo & $0.974^{* *}$ & 0.000 \\
Inseguridad sobre el futuro & 0.009 & 0.901 \\
Apoyo social y calidad del liderazgo & $0.974^{* *}$ & 0.000 \\
Doble presencia & $0.972^{* *}$ & 0.000 \\
Estima & $0.974^{* *}$ & 0.000 \\
\hline
\end{tabular}

Nota: $r=$ correlación $; p=p$ valor.

\section{DISCUSIÓN}

Según los resultados, el nivel de exposición a los riesgos psicosociales es desfavorable siendo una situación adversa y nociva para la salud de los colaboradores (Bravo \& Nazar, 2015; Moreno, 2011), esta situación genera altos niveles de estrés principalmente por las exigencias y la inseguridad en el trabajo, así como, las deficientes relaciones interpersonales evidenciadas en el inadecuado trato que recibe principalmente el colaborador obrero (Camacho \& Mayorga, 2017).

Al describir los valores categóricos de las dimensiones de la variable riesgos psicosociales se evidenció que en la mayoría de las dimensiones el nivel de exposición psicosocial es más desfavorable para la salud, es así que, en las exigencias psicológicas el $98 \%$ de colaboradores coinciden que por la cantidad de trabajo y la diferencia en las condiciones de trabajo para la mayoría (Castro, 2018) este evento es altamente riesgoso, generando enfermedades ocupacionales y accidentes de trabajo (Varela, 2014); de igual manera las variables sociodemográficas (Gil, 2012), diferencian el nivel de exposición, según sexo, son los hombres quienes mayormente están expuestos a exigencias psicológicas que las mujeres, debido a que por lo general trabajan en actividades a campo abierto en la empresa minera que demanda más cantidad de trabajo productivo, según la edad son los adultos que presentan edades por encima de los 31 años quienes consideran que la cantidad de trabajo afecta desfavorablemente la salud en comparación a los colaboradores menores de 30 años y según la condición laboral no se diferencia la exigencia, es decir, tanto los funcionarios, empleados y obreros sienten esa presión producto de la cantidad de trabajo.

En relación, al control sobre el trabajo el $56.7 \%$ precisa que el nivel de exposición es desfavorable, $28.9 \%$ 
es favorable (tienen la autonomía en la forma de realizar el trabajo) y $14.4 \%$ señala que es intermedia; al comparar la dimensión con variables como el sexo son las mujeres quienes tienen la posibilidad de controlar mejor el trabajo en comparación que los hombres, porque realizan mayormente trabajos administrativos (Tacca \& Tacca, 2019), así también, son los colaboradores funcionarios quienes controlan mejor su trabajo porque lo hacen de manera jerárquica disponiendo lo que se debe hacer en la empresa y son los colaboradores empleados y obreros quienes ejecutan esas disposiciones.

En cuanto a la inseguridad sobre el futuro en el trabajo el $80.1 \%$ considera que es desfavorable, $14.4 \%$ es de nivel intermedia y sólo el $5.5 \%$ de colaboradores indican que se sienten seguros en su trabajo (favorable), es decir, que la mayoría de colaboradores no presentan expectativas de desarrollo en la empresa, debido a que los contratos son temporales y específicos observándose que las condiciones de trabajo son desfavorables (Castro, 2018); al comparar la dimensión con el sexo, la edad y las condiciones laborales no se encontraron diferencias significativas, concluyéndose que la inseguridad sobre el futuro en el trabajo es percibida tanto por hombres y mujeres, funcionarios, empleados y obreros, y en cualquier edad, que ningún colaborador es indispensable en una empresa privada (Moreno, 2011; Gómez, Hernández, \& Méndez, 2014).

En relación al apoyo social y calidad de liderazgo según los colaboradores para el $71.6 \%$ el nivel de exposición también es desfavorable para la salud debido a que perciben que no reciben integralmente el apoyo de sus superiores y compañeros evidenciándose inadecuadas relaciones interpersonales en los colaboradores, moobing o acoso laboral, así como insatisfacción laboral, desmotivación, ansiedad, depresión (Martínez \& Hernández, 2005; Luna, Anaya, \& Ramírez, 2019); según sexo, son las mujeres quienes reciben mejor apoyo social que los hombres, según condición laboral, son los funcionarios quienes perciben mejor apoyo social en comparación con los obreros quienes perciben la ausencia de compañerismo y la falta de apoyo de sus superiores.

Según la dimensión doble presencia (respuesta simultánea a las demandas del trabajo y las actividades domésticas), el $43.8 \%$ considera que el nivel de exposición es intermedio, $29.4 \%$ es desfavorable y $26.9 \%$ considera que es favorable; para las mujeres compartir su vida laboral con las actividades del hogar es un mérito porque cumplen un doble rol, demostrando mayor organización y esfuerzo para desarrollarse en comparación a los hombres quienes consideran que esta situación es desfavorable, con respecto a la edad son los colaboradores en edad productiva (31 a 40 años) quienes demuestran mejores condiciones de adaptarse a la vida laboral con la domestica (ISTAS-CCOO, 2019).

Con referencia a la dimensión estima (trato recibido en el trabajo) el $71.6 \%$ señala que es desfavorable para la salud (no reciben un adecuado trato), 20.9\% el nivel de exposición es intermedia y $7.5 \%$ considera que es favorable. Según sexo son las mujeres quienes reciben mejor trato en el trabajo que los hombres, mientras que son los colaboradores obreros quienes reciben el peor trato en el trabajo por parte de los colaboradores empleados y funcionarios específicamente los que realizan trabajo en campo.

$\mathrm{Y}$ con respecto a las correlaciones entre la variable riesgos psicosociales y sus dimensiones la mayoría son positivas estadísticamente significativas; y en relación a las exigencias psicológicas y los riesgos psicosociales la correlación es negativa (inversa), existiendo la probabilidad que a mayor exposición psicosocial es menos favorable para la salud (existe alta probabilidad de perjudicar la salud), demostrándose que la exposición a los riesgos psicosociales en la empresa minera son desfavorables para la mayoría de los colaboradores sobre todo para el personal obrero.

\section{REFERENCIAS BIBLIOGRÁFICAS}

1. Bravo, C. \& Nazar, G. (2015). Riesgo psicosocial en el trabajo y salud en conductores de locomoción colectiva urbana en Chile. Salud de los Trabajadores, 23(2), 105-114. Recuperado de http://ve.scielo. org/scielo.php?script=sci_arttext\&pid=S1315$01382015000200004 \& \operatorname{lng}=\mathrm{es} \& \operatorname{tlng}=\mathrm{es}$

2. Camacho, A. \& Mayorga, D. (2017). Riesgos laborales psicosociales. Perspectiva organizacional, jurídica y social. Revista Prolegómenos - Derechos y Valores. 20 (40), 159-172. http://dx.doi.org/10.18359/prole.3047

3. Castro, N. (2018). Riesgos Psicosociales y Salud Laboral en Centros de Salud. Ciencia \& trabajo, 20(63), 155-159. https://dx.doi.org/10.4067/S071824492018000300155

4. COPSOQ-ISTAS. Instituto Sindical de Ambiente, Trabajo y Salud (2010). Cuestionario para la autoevaluación de riesgos psicosociales en el trabajo versión 1.5. Recuperado de http://istas.net/descargas/m_uso_ istas212.pdf

5. Frias, D. (2019). Apuntes de consistencia interna de las puntuaciones de un instrumento de medida. Universidad de Valencia. España. Disponible en: https://www.uv.es/ friasnav/AlfaCronbach.pdf

6. Gil, P. (2012). Riesgos psicosociales en el trabajo y salud ocupacional. Revista Peruana de Medicina Experimental y Salud Publica, 29(2), 237-241. Recuperado de http://www.scielo.org.pe/scielo.php? script=sci arttext\&pid=S1726-46342012000200012\&lng=es\&tln $\mathrm{g}=\mathrm{es}$

7. Gómez, P., Hernández, J. \& Méndez, M. (2014). Factores de Riesgo Psicosocial y Satisfacción Laboral en una Empresa Chilena del Área de la Minería. Ciencia \& trabajo, 16(49), 9-16. https://dx.doi.org/10.4067/ S0718-24492014000100003

8. ISTAS-CCOO. Instituto Sindical de Trabajo, Ambiente y Salud. (2019). Relación de las exposiciones psicosociales con la edad: trabajadores jóvenes y mayores y necesidades de conciliación. Recuperado de https://istas.net/sites/default/files/2019-04/ Informe $\% 202019-\% 20 \mathrm{Jo}_{0} \mathrm{CC} \% 81$ venes $\% 2 \mathrm{C} \% 20$ Mayores\%2C\%20Conciliacio\%CC\%81n\%202.pdf

9. Ley $N^{\circ}$ 29783. Ley de Seguridad y Salud en el Trabajo.

10. Luna, E., Anaya, A. \& Ramírez, E. (2019). Diagnóstico de las percepciones de los factores de riesgo psicosociales en el trabajo del personal de una industria manufacturera. Estudos de Psicologia (Campinas), 36, 1-10. https://doi.org/10.1590/1982$0275201936 \mathrm{e} 180148$

11. Martínez, S. \& Hernández, A. (2005). Necesidad 
de estudios y legislación sobre factores psicosociales en el trabajo. Revista Cubana de Salud Pública, 31(4) Recuperado de http://scielo. sld.cu/scielo.php?script $=$ sci_arttext\&pid $=$ S086434662005000400012\&lng=es\&tlng=es

12. Ministerio de Energía y Minas (2019). Estadística de enfermedades ocupacionales en minería. Recuperado de http://www.minem.gob.pe/_estadistica. php?idSector $=1 \&$ idEstadistica $=10187$

13. Ministerio del Trabajo y Promoción del Empleo (2019). Boletín estadístico mensual. Notificaciones de accidentes de trabajo, incidentes peligrosos y enfermedades ocupacionales. Recuperado de https:// cdn.www.gob.pe/uploads/document/file/536368/ Bolet\%C3\%ADn Notificaciones_DICIEMBRE 2019. pdf

14. Moreno, B. (2011). Factores y riesgos laborales psicosociales: conceptualización, historia y cambios actuales. Medicina y Seguridad del Trabajo, 57 (Supl. 1), 4-19. https://dx.doi.org/10.4321/S0465546X2011000500002

15. Tacca, D. \& Tacca, A. (2019). Factores de riesgos psicosociales y estrés percibido en docentes universitarios. Propósitos y Representaciones, 7 (3), 323 - 353. http://dx.doi.org/10.20511/pyr2019. v7n3.304

16. Varela, L. (2014). Riesgos psicosociales presentes en el trabajo. Ciencia \& trabajo, 16 (50), a01. https://dx.doi. org/10.4067/S0718-24492014000200001

17. Yazici, B., \& Yolacan, S. (2007). A comparison of various tests of normality. Journal of Statistical Computation and Simulation, 77(2), 175-183. http:// dx.doi. org/10.1080/10629360600678310 